\title{
Increased serum amyloid $A$ and its association with autoantibodies, acute phase reactants and disease activity in patients with rheumatoid arthritis
}

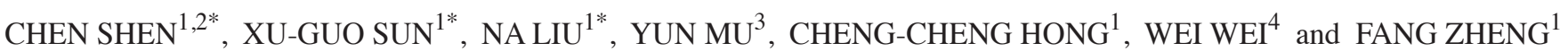 \\ ${ }^{1}$ Department of Clinical Immunology, School of Laboratory Medicine, Tianjin Medical University, Tianjin 300203; \\ ${ }^{2}$ Department of Medical Laboratory, Jining No. 1 People's Hospital, Jining, Shandong 272011; \\ ${ }^{3}$ Department of Medical Laboratory, Tianjin Hospital of Children, Tianjin 300074; ${ }^{4}$ Department of \\ Rheumatology, General Hospital, Tianjin Medical University, Tianjin 300052, P.R. China
}

Received January 25, 2014; Accepted October 1, 2014

DOI: $10.3892 / \mathrm{mmr} .2014 .2804$

\begin{abstract}
Determination of disease activity in patients with rheumatoid arthritis (RA) has become an important component for RA management. The aim of the present study was to investigate the association between circulating levels of serum amyloid A (SAA) and disease activity in RA patients. The types of disease and the respective number of patients enrolled in the present study were as follows: RA, 88; osteoarthritis (OA), 54; systemic lupus erythematosus (SLE), 43; and other autoimmune diseases, 30 , as well as 50 healthy controls (HC). SAA levels were measured using an ELISA assay and western blot analysis was used to detect serum SAA levels. The correlations between SAA levels and disease activity score for 28 joints (DAS28), erythrocyte sedimentation rate (ESR) and C-reactive protein (CRP), respectively, were evaluated; in addition, the presence and absence of rheumatoid factor (RF) and anti-cyclic citrullinated peptide antibody (anti-CCP) were detected in respect to SAA levels. The results of the present study demonstrated that serum levels of SAA in RA patients were significantly increased compared to those of the OA, SLE, others and HC patients $(\mathrm{P}<0.05)$. SAA levels were found to be positively correlated with DAS28, ESR and CRP levels $\left(\mathrm{R}^{2}=0.6174,0.4422\right.$ and 0.3919 , respectively). In addition, anti-CCP was not correlated with DAS28 $\left(\mathrm{R}^{2}=0.0154\right)$. Furthermore, increased SAA levels were detected in patients with positive anti-CCP compared with those in anti-CCP negative subjects $(\mathrm{P}<0.01)$. In conclusion, the results of the
\end{abstract}

Correspondence to: Professor Fang Zheng, Department of Clinical Immunology, School of Laboratory Medicine, Tianjin Medical University, 1 Guangdong Road, Tianjin 300203, P.R. China

E-mail: Fangzheng@tmu.edu.cn

*Contributed equally

Key words: serum amyloid A, rheumatoid arthritis, disease activity, acute phase protein present study provided further evidence for possible roles of SAA in RA, which indicated that it may be a useful biomarker for assessing disease severity and may provide additional information about disease activity.

\section{Introduction}

Rheumatoid arthritis (RA) is the most prevalent chronic inflammatory disease, which is characterized by synovial proliferation, progressive damage disability and systemic complications (1). The severity of the disease is associated with inflammation intensity and joint damage (2). The current treatment strategy involves aggressive therapy, which is selected using an assessment of disease activity in pursuit of clinical remission (3). Therefore, determination of disease activity in RA patients has become an important component for RA management.

Several indices have been used to assess RA disease activity based on the clinical manifestations, laboratory and physical measurements. These indices include the disease activity score for 28 joints (DAS28), rheumatoid arthritis disease activity index (RADAI) and clinical disease activity index (CDAI) (3). The majority of RA indices rely on swollen and tender joint counts as well as patient-reported outcomes, of which DAS28 is widely used to monitor and assess disease activity in clinical trials and routine patient care $(4,5)$. However, significant variability occurs between different observers, which influences results. Additional laboratory tests are therefore required in order to augment clinical assessment. Erythrocyte sedimentation rates (ESR) and C-reactive protein (CRP) are both incorporated into disease activity assessments in RA patients (6). ESR and CRP are important inflammatory markers; however, they are reported to be increased with aging and anemia (7). The presence of rheumatoid factor (RF) and anti-cyclic citrullinated peptide antibody (anti-CCP) have been associated with disease severity and joint erosions, which are included in the criteria for RA (9); however, RF and anti-CCP were reported to have little correlation with disease activity (10). Therefore, the elucidation of further surrogate biomarkers for RA disease activity assessment is critical. 
Table I. Clinical characteristics of patients in each group.

\begin{tabular}{llllcc}
\hline Group & $\begin{array}{c}\text { Gender } \\
(\mathrm{F} / \mathrm{M})\end{array}$ & $\begin{array}{c}\text { Age } \\
\text { (years) }\end{array}$ & $\begin{array}{c}\text { Erythrocyte } \\
\text { sedimentation } \\
\text { rate }(\mathrm{mm} / \mathrm{h})\end{array}$ & $\begin{array}{c}\text { C-reactive } \\
\text { protein }(\mathrm{mg} / \mathrm{l})\end{array}$ \\
\hline RA & $\mathrm{n}$ & $65 / 23$ & $58 \pm 12$ & $49.06 \pm 13.79^{\mathrm{a}, \mathrm{b}}$ & $41.42 \pm 32.83^{\mathrm{a}, \mathrm{b}}$ \\
SLE & 88 & $31 / 12$ & $53 \pm 17$ & $41.80 \pm 17.30$ & $35.74 \pm 31.61$ \\
Others & 43 & $25 / 5$ & $51 \pm 13$ & $45.06 \pm 11.43$ & $29.82 \pm 23.37$ \\
OA & 30 & $42 / 12$ & $56 \pm 16$ & $23.26 \pm 18.51$ & $28.55 \pm 21.05$ \\
HC & 54 & $38 / 12$ & $52 \pm 8$ & $12.50 \pm 11.90$ & $5.02 \pm 3.11$ \\
\hline
\end{tabular}

Values are presented as the mean \pm standard deviation. ${ }^{\mathrm{a}} \mathrm{P}<0.05 \mathrm{RA}$ vs. $\mathrm{OA},{ }^{\mathrm{b}} \mathrm{P}<0.05 \mathrm{RA}$ vs. HC. RA, rheumatoid arthritis; SLE, systemic lupus erythematosus; Others, other autoimmune diseases; OA, osteoarthritis; HC, healthy control; F/M, female/male.

Serum amyloid A (SAA) is an acute phase protein, $\sim 12 \mathrm{kDa}$ in size. SAA is primarily synthesized by hepatocytes and secreted from certain extrahepatic sites in chronic inflammatory diseases, including Alzheimer's disease and RA (10). The circulatory SAA concentration was reported to be significantly increased during acute phase responses, including trauma and infection (11). SAA was also shown to exhibit important immunological functions in the process of inflammation; for example, SAA may be a chemokine for immune cells, including T lymphocytes, neutrophils and mast cells. SAA was also found to stimulate the synthesis of several pro-inflammatory cytokines, including interleukin-1 (IL-1) and tumor necrosis factor (TNF) (12). Previous studies have indicated that SAA positively correlated with disease activity in ankylosing spondylitis (AS) (13), juvenile idiopathic arthritis (JIA) (14) and polymyalgia rheumatica (PMR) (15). The aim of the present study was to investigate the association between circulating levels of SAA and disease activity in RA patients.

\section{Materials and methods}

Patients and samples. The types of disease and the respective number of patients that serum samples were obtained from were as follows: RA, 88; osteoarthritis (OA), 54; systemic lupus erythematosus (SLE), 43; and other autoimmune diseases, 30 (Sjogren's syndrome, 15; ankylosing spondylitis, 5; systemic scleroderma, 5; spondyloarthropathy, 2; psoriatic arthritis, 2; and chondritis, 1), as well as 50 age and gender-matched healthy control subjects (HC) (Table I). Matched synovial fluid (SF) and synovial membrane (SM) samples were obtained from $20 \mathrm{RA}$ and $16 \mathrm{OA}$ patients undergoing arthroscopic biopsies and joint replacement surgeries. All patients with RA fulfilled the American College of Rheumatology (ACR; Atlanta, GA, USA) criteria for RA; in addition, all other patients enrolled in the present study fulfilled their corresponding diagnostic criteria. The present study was approved by the Medical Ethics and Human Clinical Trial Committee of Tianjin Medical University (Tianjin, China).

Sample preparation. All samples, including clotted serum and SF, were centrifuged at $1,425.6 \mathrm{~g}$ for $10 \mathrm{~min}$, immediately aliquoted and stored at $-80^{\circ} \mathrm{C}$. All samples were only allowed to be thawed once.

Determination of SAA levels in sera and SF using ELISA. The concentration of SAA in serum and SF was detected using a sandwich ELISA (human SAA ELISA kit; Xinle Biology Co., Ltd., Shanghai, China) according to the manufacturer's instructions. Optical density (OD) values of each well were measured using an ELISA plate reader (Multiskan MK3; Thermo Scientific, Waltham, MA, USA) at $450 \mathrm{~nm}$.

Western blot analysis of SAA expression in sera. All serum samples were diluted and denatured at $95^{\circ} \mathrm{C}$ for 5 min following the addition of loading buffer (Zhaoran Biology Co., Ltd., Shanghai, China). Serum proteins were separated using SDS-PAGE (Zhaoran Biology Co., Ltd.) and subsequently transferred onto the polyvinylidene difluoride (PVDF; Yongyuan Metal Co., Ltd., Suzhou, China) membrane for $1 \mathrm{~h}$ at $250 \mathrm{~mA}$. The membrane was then blocked for $1 \mathrm{~h}$ at room temperature in $5 \%$ skimmed milk/tris-buffered saline with Tween 20 (TBST; $20 \mathrm{mM}$ Tris-HCl, pH 7.6; $137 \mathrm{mM} \mathrm{NaCl}$; and $0.05 \%$ Tween 20) and incubated with rabbit anti-human SAA polyclonal antibodies (Abcam, Cambridge, UK) for $1 \mathrm{~h}$ at room temperature (1:2,000 in 5\% skimmed milk/TBST). The membrane was washed with TBST three times for 30 min each and then incubated with horseradish peroxidase (HRP)-conjugated goat anti-rabbit immunoglobulin (Ig)G (Bioword Technology, Inc., St. Louis Park, MN, USA) for $1 \mathrm{~h}$ (1:1,000 in 5\% skimmed milk/TBST). Following washing, proteins were detected using a Pierceenhanced chemiluminescence system (Solarbio Bioscience and Technology Co., Ltd, Shanghai, China).

Immunohistochemistry. Synovial tissue sections were fixed in acetone for $10 \mathrm{~min}$, incubated with primary rabbit polyclonal antibodies against human SAA (1:1,200; Abcam) for $1 \mathrm{~h}$ at $37^{\circ} \mathrm{C}$. An isotype-matched mouse monoclonal antibody (1:500; Xinle Biology Co., Ltd.) was used as the negative control. HRP-conjugated goat anti-rabbit IgG secondary antibodies $(1: 1,00)$ were then added and incubated for $1 \mathrm{~h}$. Color was developed in solutions containing diaminobenzadine-tetrahydrochloride (Sigma-Aldrich, St. Louis, MO, USA) and $0.5 \% \mathrm{H}_{2} \mathrm{O}_{2}$ in phosphate-buffered saline ( $\mathrm{pH}$ 7.6; Zhaoran Biology Co., Ltd.). Slides were then counter-stained with 
Table II. Serum levels of serum amyloid A in each group.

\begin{tabular}{lcccc}
\hline Group & $\mathrm{n}$ & $\begin{array}{c}\text { Mean } \pm \text { standard } \\
\text { deviation }(\mathrm{mg} / \mathrm{l})\end{array}$ & F-value & P-value \\
\hline RA & 88 & $6.15 \pm 3.27^{\mathrm{a}, \mathrm{b}}$ & & \\
SLE & 43 & $2.54 \pm 0.31$ & & \\
Others & 30 & $1.55 \pm 0.98$ & 5.549 & 0.006 \\
OA & 54 & $1.42 \pm 0.97$ & & \\
HC & 50 & $1.45 \pm 0.72$ & & \\
\hline
\end{tabular}

${ }^{\mathrm{a}} \mathrm{P}<0.05 \mathrm{RA}$ vs. OA, ${ }^{\mathrm{b}} \mathrm{P}<0.05 \mathrm{RA}$ vs. HC. RA, rheumatoid arthritis; SLE, systemic lupus erythematosus; Others, other autoimmune diseases; OA, osteoarthritis; HC, healthy control.

hematoxylin and mounted (Zhaoran Biology Co., Ltd.). SAA levels were determined through quantification of the number of positive-staining cells/high-power field.

Determination of disease activity. DAS28-ESR, a validated scoring system for predicting disease activity, was employed in order to calculate disease activity using the following formula: DAS28-ESR=0.56xsqrt(number of tender joints) $+0.28 x$ sqrt(number of swollen joints) + 0.70xLn(ESR) + 0.014xvisual analogue scale (VAS).

VASs are a straight horizontal line of fixed length, usually $100 \mathrm{~mm}$. The ends are defined as the extreme limits of the measured parameter (e.g. symptom, pain and health) orientated from the left (worst) to the right (best). ESR was measured in $\mathrm{mm} / \mathrm{hr}$ and VAS was measured in $\mathrm{mm}$. The range of the tests varies from 0 to 10 .

Clinical and laboratory measurements. General clinical data, including age, gender, disease duration, number of swollen joints and number of tender joints, were collected. Laboratory data were obtained as follows: ESR was measured using the Westergren method (16); CRP was examined using the immunonephelometry method (16); RF-IgA and RF-IgG were measured in serum using rate nephelometry (Immage ${ }^{\circledR}$ Immunochemistry system; Beckman Coulter, Brea, CA, USA). The anti-CCP2 antibody was tested using a second-generation ELISA kit (Shanghai Fuchun-Zhongnan Biotech Co., Ltd, Shanghai, China).

Statistical analysis. Data are presented as the mean \pm standard deviation. Data were processed using SPSS 11.0 (SPSS Inc., Chicago, IL, USA). Differences among groups were analyzed using a one-way analysis of variance (ANOVA). Comparisons between two groups were analyzed using a Student-Newman-Keuls test or Student's t-test. Correlations were determined using the Spearman rank correlation coefficients. $\mathrm{P}<0.05$ was considered to indicate a statistically significant difference between values.

\section{Results}

Clinical characters of the participants. Detailed clinical characteristics of all participants are shown in Table I. The data

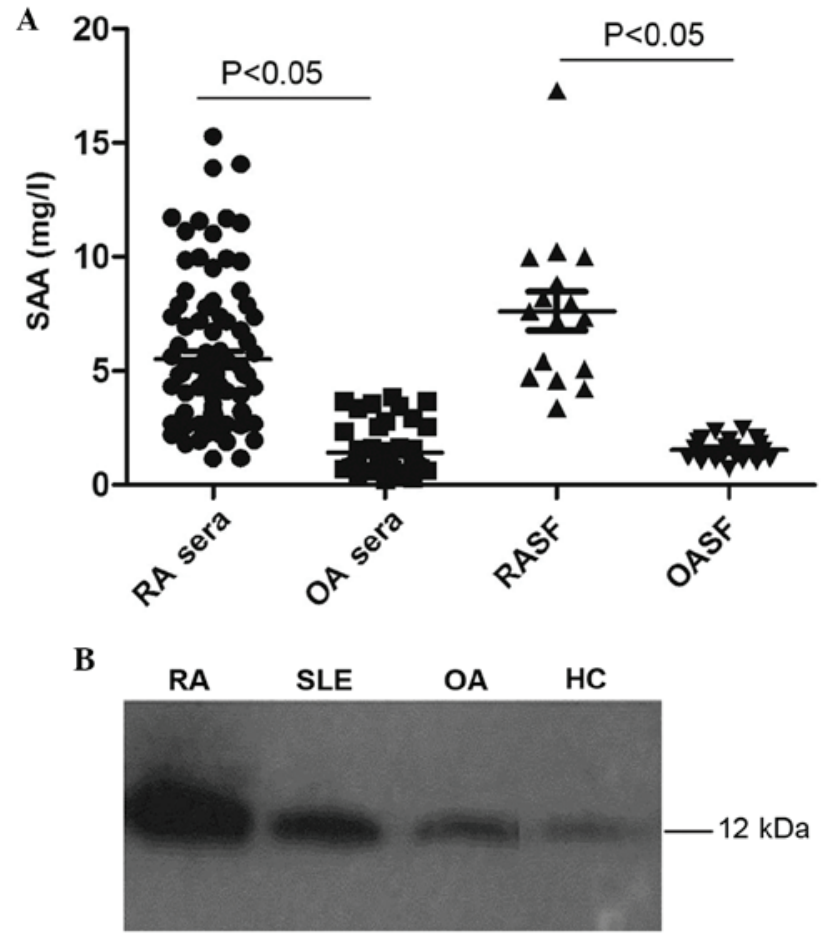

Figure 1. Increased levels of SAA in the serum and synovial fluid of RA patients. (A) ELISA analysis of serum SAA in RA and OA patients $(n=88$ and 54 , respectively), as well as SF SAA levels in RA and OA patients ( $n=16$ and 20, respectively). (B) Representative western blot analysis of SAA expression in the serum of RA, SLE, OA and HC patients. SAA, serum amyloid A; SF, synovial fluid; RA, rheumatoid arthritis; OA, osteoarthritis; SLE, systemic lupus erythematosus; HC, healthy control.

demonstrated no significant differences in gender balance or average age of patients among the groups $(\mathrm{P}>0.05)$. However, the results showed that ESR and CRP were significantly increased in the RA group compared to those in the $\mathrm{HC}$ and OA groups $(\mathrm{P}<0.05)$.

Increased expression of SAA inpatients with RA. An ELISA assay was performed in order to detect serum levels of SAA. Data from five groups were analyzed using one-way ANOVA (Table II). The results revealed that there was a statistically significant difference among the five groups $(\mathrm{F}=5.549 ; \mathrm{P}<0.05)$. Further statistical analysis, using the Student-Newman-Keuls test, showed that serum SAA levels in RA $(6.15 \pm 3.27 \mathrm{mg} / \mathrm{l})$ were significantly increased compared to those of the SLE, other autoimmune diseases, OA and HC groups. However, no significant differences were identified among the other groups.

Further ELISA assays were used to detect levels of SAA in SF. As shown in Fig. 1A, SAA levels were significantly increased in the $\mathrm{SF}$ of the RA group $(7.63 \pm 3.39 \mathrm{mg} / \mathrm{l})$ compared to those of the OA group $(1.54 \pm 0.48 \mathrm{mg} / \mathrm{l} ; \mathrm{P}<0.05)$.

Western blot analysis of serum SAA levels revealed that SAA protein expression levels were markedly increased in RA patients compared with those of individuals in the SLE, OA and $\mathrm{HC}$ groups (Fig. 1B).

Immunohistochemical analysis of SAA expression in the SM. Hematoxylin staining revealed high levels of SAA in all tissue samples from RA patients. The histological distribution of SAA 

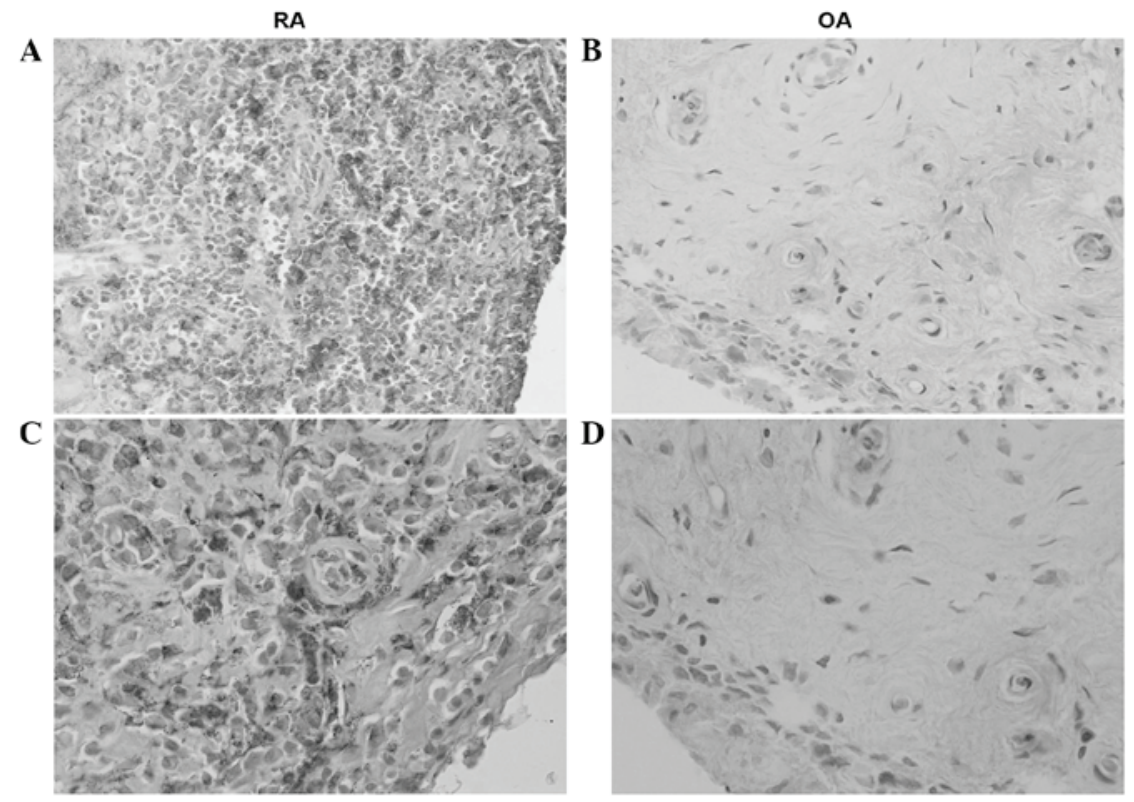

Figure 2. Increased SAA expression in the SM of RA patients. SM sections from (A and C) RA ( $n=7$ ) and (B and D) OA patients (n=12) were stained with anti-SAA antibodies. A brown stain indicated cells positive for SAA. In RA patients SAA expression was observed in synovial vascular endothelial cells, perivascular areas and inflammatory cells. Minimal staining of perivascular areas and fibroblast-like synovial cells was observed in OA tissue (magnification: A and B, x200; C and D, x400). SAA, serum amyloid A; SM, synovial membrane; RA, rheumatoid arthritis; OA, osteoarthritis.

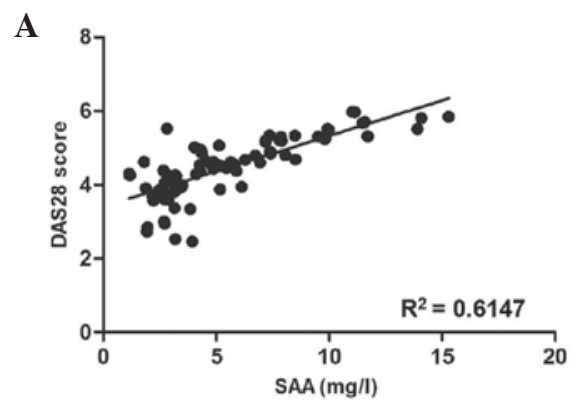

D

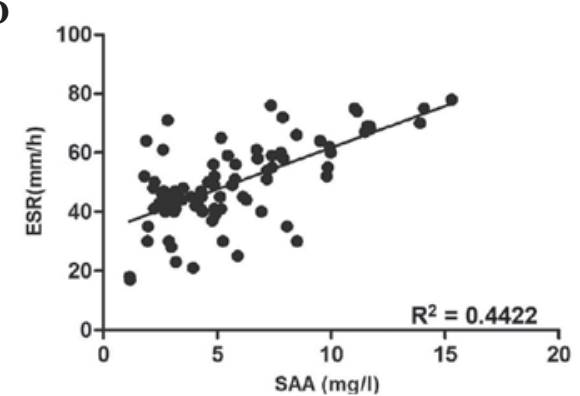

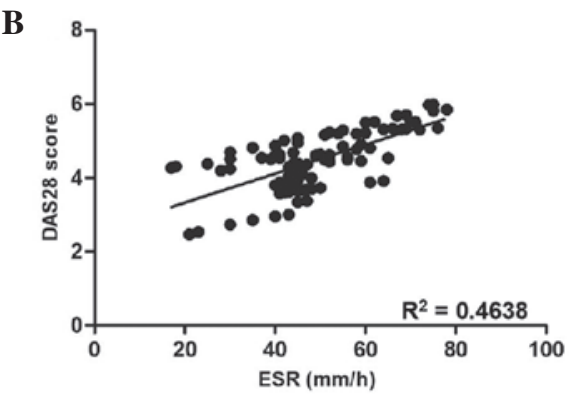
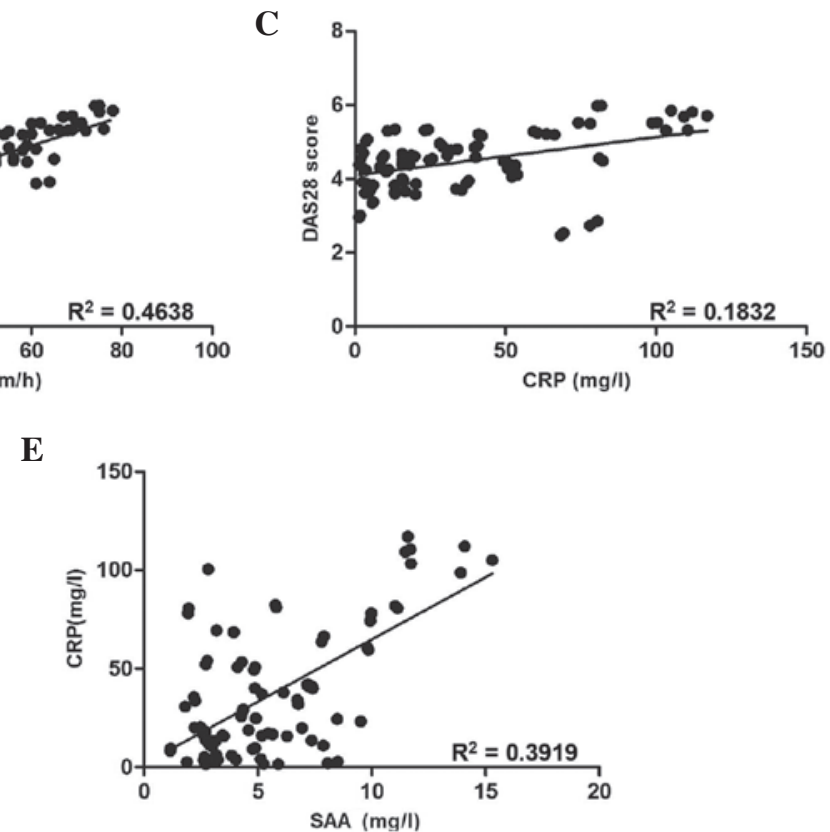

Figure 3. Scatter plots demonstrating the correlations of serum SAA levels with rheumatoid arthritis disease activity: (A) Serum SAA levels with DAS28 score $\left(\mathrm{R}^{2}=0.6147\right.$; $\left.\mathrm{P}<0.001\right)$; (B) ESR with DAS28 score $\left(\mathrm{R}^{2}=0.4638\right.$; $\left.\mathrm{P}<0.001\right)$; (C) $\mathrm{CRP}$ with DAS28 score $\left(\mathrm{R}^{2}=0.1832 ; \mathrm{P}=0.004\right)$; (D) serum SAA levels with ESR $\left(\mathrm{R}^{2}=0.4422 ; \mathrm{P}<0.001\right)$; and $(\mathrm{E})$ serum SAA levels with $\mathrm{CRP}\left(\mathrm{R}^{2}=0.3919 ; \mathrm{P}<0.001\right)$. SAA, serum amyloid A; DAS28, disease activity score for 28 joints; $\mathrm{ESR}$, erythrocyte sedimentation rates; $\mathrm{CRP}, \mathrm{C}$-reactive protein; $\mathrm{R}^{2}$, correlation coefficient.

was located in synovial lining and sublining layers; of note, in fibroblast-like synovial cells (FLSs), inflammatory cells, vascular endothelial cells and perivascular areas. However, staining for SAA in tissue samples from patients in the OA group was markedly weaker in perivascular areas and FLSs (Fig. 2).

Serum SAA levels correlate with RA disease activity, ESR and $C R P$. Spearman's rank correlation analyses were performed to assess correlations between the levels of SAA in RA patients' sera and DAS28 (Fig. 3A). A significant correlation was observed between SAA levels in sera and DAS28 in RA patients $\left(\mathrm{R}^{2}=0.6147 ; \mathrm{P}<0.001\right)$, therefore indicating that serum levels of SAA in RA patients were positively correlated with disease activity. In addition, the correlation of serum SAA levels with other serologic biomarkers was assessed. As shown in Fig. 3B and C, ESR and CRP levels were demonstrated to be 
A

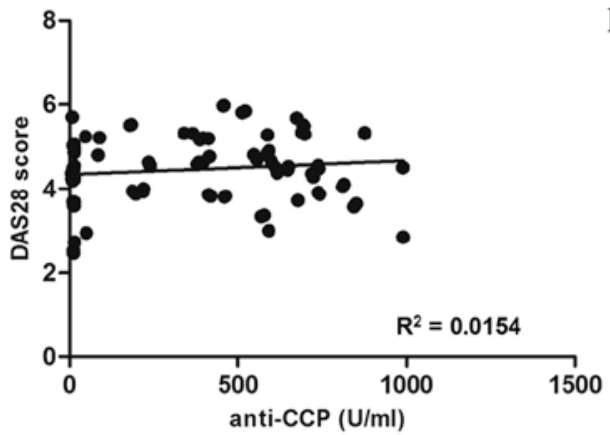

C

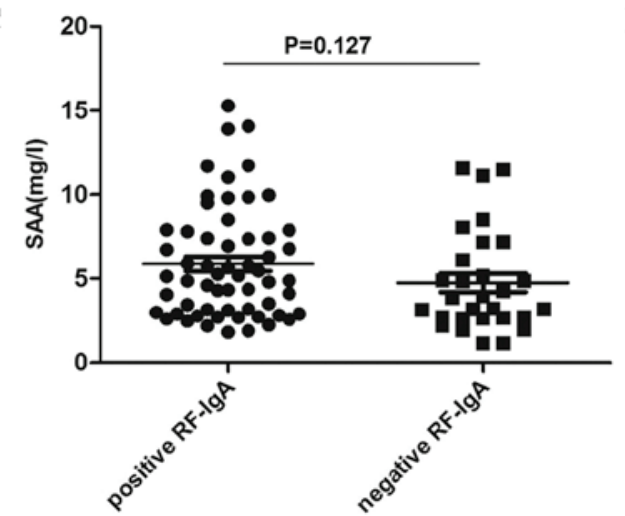

B

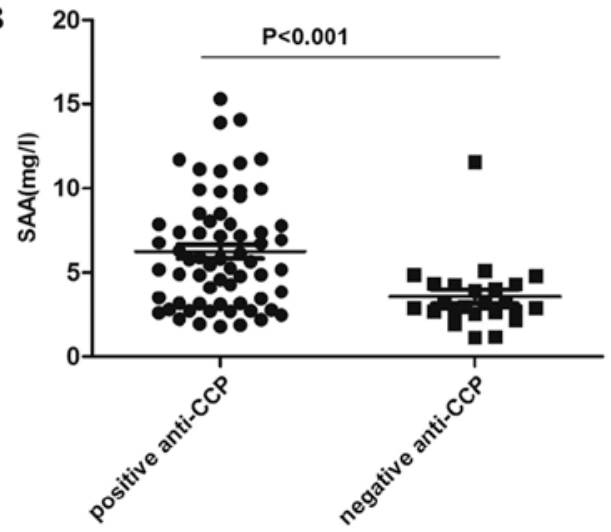

D

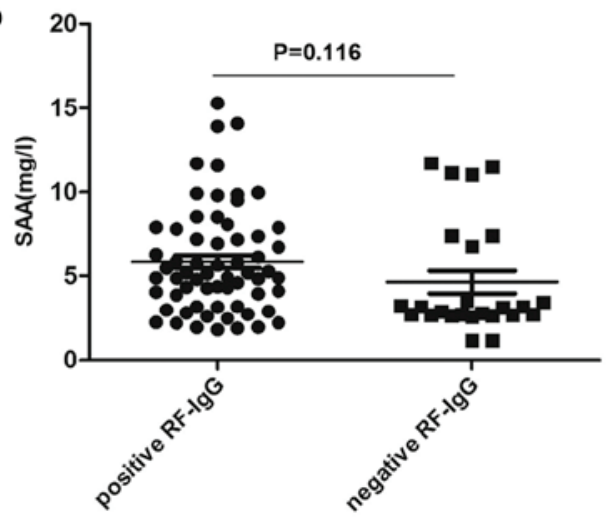

Figure 4. Levels of SAA in RA patients positive or negative for auto antibodies. (A) Comparison of anti-CCP and DAS28 scores showed no significant correlation $\left(\mathrm{R}^{2}=0.0154, \mathrm{P}=0.250\right)$. (B) SAA levels in anti-CCP positive were significantly increased compared to those of anti-CCP-negative $\mathrm{RA}$ patients $(\mathrm{P}<0.001)$. Comparison of SAA levels in (C) RF-IgA-positive or -negative or (D) RF-IgG-positive or -negative RA patients showed no significant differences between groups ( $\mathrm{P}=0.127$ and 0.116 , respectively). SAA, serum amyloid A; DAS28, disease activity score for 28 joints; $\mathrm{CCP}$, cyclic citrullinated peptide; RF, rheumatoid factor; Ig, immunoglobulin.

positively correlated with DAS28 $\left(\mathrm{R}^{2}=0.4638, \mathrm{P}<0.001\right.$; and $\mathrm{R}^{2}=0.1832, \mathrm{P}=0.004$, respectively). Furthermore, SAA levels were found to be positively correlated with $\operatorname{ESR}\left(R^{2}=0.4422\right.$; $\mathrm{P}<0.001)$ and $\mathrm{CRP}\left(\mathrm{R}^{2}=0.3919 ; \mathrm{P}<0.001\right)$ (Fig. 3D and $\left.\mathrm{E}\right)$.

Association between SAA and autoantibodies in RA. As shown in Fig. 4A, no significant correlation was identified between serum anti-CCP levels and DAS28 $\left(\mathrm{R}^{2}=0.0154 ; \mathrm{P}=0.250\right)$. However, RA patients positive for anti-CCP showed significantly increased levels of SAA compared to those of patients negative for anti-CCP $(6.88 \pm 3.36$ and $4.20 \pm 2.02 \mathrm{mg} / \mathrm{l}$, respectively; $\mathrm{P}<0.001$ ) (Fig. 4B). In addition, RA patients positive for RF-IgA demonstrated a non-significant increase in SAA levels compared to those of IgA-negative patients $(6.53 \pm 3.33$ and $5.41 \pm 3.00 \mathrm{mg} / 1$, respectively; $\mathrm{P}=0.127$ ) (Fig. 4C). Identical conclusions were drawn from the results for RF-IgG-positive and -negative groups $(6.50 \pm 3.17$ and $5.29 \pm 3.37 \mathrm{mg} / \mathrm{l}$; $\mathrm{P}=0.116$ ) (Fig. 4D).

\section{Discussion}

The aim of the present study was to investigate the association between circulating levels of SAA and disease activity in RA patients. The results showed that SAA levels in RA patients were significantly increased compared to those of the control and other disease groups. In addition, the results of the present study demonstrated a significant correlation between
SAA levels and DAS28 as well as other validated disease activity measures; this therefore indicated that SAA was a useful indicator of RA disease activity.

Previous studies have reported that serum SAA levels were elevated in RA patients compared with those of SLE patients (17-19). The results of the present study were consistent with these findings, demonstrating that serum SAA levels were significantly increased not only compared with the SLE group but also with patients with other autoimmune diseases $\mathrm{OA}$ and HC; therefore, this implied its potential value for monitoring RA disease activity. The results of the present study were also consistent with a study by de Seny et al (20), which showed significantly elevated plasma SAA levels in RA. SAA is produced via the signaling pathways of pro-inflammatory cytokines, including interleukin (IL)-6 and IL-8 (12). During acute phase responses, including inflammation and infection, SAA concentrations may be elevated as much as 1,000 fold (7). A study by Metawi et al (21) showed that IL-17, secreted by Th17 cells, acted as an indicator of RA disease activity. Another study confirmed that SAA induced the production of CCL20, which has a role in the recruitment of Th17 cells to the inflammatory sites; moreover, the expression of CCL20 was reported to be upregulated by IL-17 (22). These results also suggested the possible role of SAA in disease activity of RA. In the present study, western blot analysis was used in order to determine the expression of SAA in sera, the result of which was consistent with that of the ELISA assays performed. Of 
note, SAA monomers were located in sera, whereas other forms, including dimers or combined SAA, were not observed.

Furthermore, the results of the present study showed that SAA levels were significantly increased in the synovial fluid and synovial membrane of RA patients compared to those of OA patients; these results were in accordance with previous studies $(20,23)$. Radiographic data revealed that RA patients enrolled in the present study showed signs of cartilage destruction, coarse and erosion (data not shown). It is well-established that SAA in synovial fluid is primarily produced by local joint synoviocytes; in addition, it was reported that SAA messenger RNA was upregulated in RA synovium (17). Okamoto et al (24) demonstrated that SAA activated nuclear factor- $\kappa \mathrm{B}$ via binding to the advanced glycation end-product (RAGE) receptor in rheumatoid synovial fibroblasts, which subsequently promoted the expression of pro-inflammatory cytokines IL-6 and IL-8. These results therefore suggested that SAA may have a pathophysiological role in the pathogenesis of RA.

In the present study, a significant correlation was identified between SAA and established disease activity measures. DAS28 is the most commonly used index for assessing disease activity levels in RA patients (25). The results of the present study indicated that SAA correlated with DAS28 $\left(\mathrm{R}^{2}=0.6147\right)$ in RA. Connolly et al (26) reported that SAA levels correlated with RA disease activity, as measured using swollen joint counts $\left(\mathrm{R}^{2}=0.26 ; \mathrm{P}=0.048\right)$. In the present study, $\mathrm{RA}$ disease activity was calculated using DAS28, which included swollen joint counts, tender joint counts, ESR and VAS. In addition, the patients recruited to the present study were in the stage of acute inflammation with no therapy; by contrast. The patients recruited by Connolly et al were at 0-3 months into anti-tumor necrosis factor a (anti-TNFa) therapy. The results of the present study demonstrated a significant positive correlation between SAA and ESR, CRP as well as disease activity in RA patients. ESR and CRP are both effective indicators of disease activity. It was previously reported that SAA and CRP were primarily produced in the liver; however, serum levels of SAA depend on a greater amount of pro-inflammatory cytokines compared with CRP (27), this therefore suggested that SAA may be a more sensitive biomarker than CRP for reflecting disease activity.

Autoantibodies were suggested to be important for RA diagnosis and prognosis. Anti-CCP is highly sensitive and specific for RA diagnosis, which has been associated with the presence of severe bone erosion (28). In the present study, an association was identified between elevated SAA levels and anti-CCP-positive patients, indicating that SAA may be associated with bone erosion. Increased levels of SAA in RF-IgA and RF-IgG-positive groups were observed; however, these results were not significant compared to those of the negative group. These results may be explained by the low specificity of $\mathrm{RF}$, since RF can also be detected in other infectious diseases, autoimmune diseases and even healthy people.

In conclusion, the results of the present study demonstrated that increased circulating and local SAA levels were significantly correlated with the degree of RA disease activity. These results provided further evidence for the pathological role of SAA in RA, which may be a useful biomarker in the assessment of disease severity and response to therapy. Further studies are required prior to the use of SAA as an indicator of RA disease activity in a clinical environment, for which a larger scale of samples is recommended.

\section{Acknowledgements}

The present study was supported by a grant from the Specialized Research Fund for the Doctoral Program of Higher education funded by the Ministry of Education (no. 20101202110008) and the Natural Science Foundation of Tianjin (no. 14JCYBJC25600). The authors would like to thank all subjects for their participation in the present study.

\section{References}

1. Scott DL, Wolfe F and Huizinga TW: Rheumatoid arthritis. Lancet 376: 1094-1108, 2010.

2. McInnes IB and Schett G: The pathogenesis of rheumatoid arthritis. N Engl J Med 365: 2205-2219, 2011.

3. Emery P: Evidence supporting the benefit of early intervention in rheumatoid arthritis. J Rheumatol 66: 3-8, 2002

4. Fransen J and van Riel PL: The disease activity score and the EULAR response criteria. Clin Exp Rheumatol 23 (5 Suppl 39): S93-S99, 2005.

5. Aletaha D, Landewe R, Karonitsch T, et al; EULAR, ACR: Reporting disease activity in clinical trials of patients with rheumatoid arthritis: EULAR/ACR collaborative recommendations. Arthritis Rheum 59: 1371-1377, 2008.

6. Matsui T, Kuga Y, Kaneko A, et al: Disease Activity Score 28 (DAS28) using C-reactive protein underestimates disease activity and overestimates EULAR response criteria compared with DAS28 using erythrocyte sedimentation rate in a large observational cohort of rheumatoid arthritis patients in Japan. Ann Rheum Dis 66:1221-1226, 2007.

7. Da Mota LM, dos Santos Neto LL, Burlingame R, et al: Laboratory characteristics of a cohort of patients with early rheumatoid arthritis. Rev Bras Rheumatol 50: 375-388, 2010.

8. Aletaha D, Neogi T, Silman AJ, et al: 2010 Rheumatoid arthritis classification criteria: an American College of Rheumatology/European League Against Rheumatism collaborative initiative. Arthritis Rheum 62: 2569-2581, 2010.

9. Papadopoulos NG, Tsiaousis GZ, Pavlitou-Tsiontsi A, Giannakou A and Galanopoulou VK: Does the presence of anti-CCP autoantibodies and their serum levels influence the severity and activity in rheumatoid arthritis patients? Clin Rev Allergy Immunol 34: 11-15, 2008.

10. Mullan RH, Bresnihan B, Golden-Mason L, et al: Acute-phase serum amyloid A stimulation of angiogenesis, leukocyte recruitment, and matrix degradation in rheumatoid arthritis through an NF- $\kappa \mathrm{B}$-dependent signal transduction pathway. Arthritis Rheum 54: 105-114, 2006.

11. Dong Z, Wu T, Qin W, et al: Serum amyloid A directly accelerates the progression of atherosclerosis in apolipoprotein E-deficient mice. Mol Med 17: 1357-1364, 2011.

12. Eklund KK, Niemi K and Kovanen PT: Immune functions of serum amyloid A. Crit Rev Immunol 32: 335-348, 2012.

13. Jung SY, Park MC, Park YB and Lee SK: Serum amyloid a as a useful indicator of disease activity in patients with ankylosing spondylitis. Yonsei Med J 48: 218-224, 2007.

14. Cantarini L, Giani T, Fioravanti A, et al: Serum amyloid A circulating levels and disease activity in patients with juvenile idiopathic arthritis. Yonsei Med J 53: 1045-1048, 2012.

15. Shimojima Y, Matsuda M, Gono T, Ishii W and Ikeda S: Serum amyloid $\mathrm{A}$ as a potent therapeutic marker in a refractory patient with polymyalgia rheumatica. Intern Med 44: 1009-1012, 2005.

16. Ferrari R, Tanni SE, Caram LM, Corrêa C, Corrêa CR, et al: Three-year follow-up of interleukin 6 and C-reactive protein in chronic obstructive pulmonary disease. Respir Res 20: 14-24, 2013.

17. O'Hara R, Murphy EP, Whitehead AS, FitzGerald O and Bresnihan B: Acute-phase serum amyloid A production by rheumatoid arthritis synovial tissue. Arthritis Res 2: 142-144, 2000

18. Momohara S, Okamoto $\mathrm{H}$ and Yamanaka H: Chondrocyte of rheumatoid arthritis serve as a source of intra-articular acute-phase serum amyloid A protein. Clin Chim Acta 398: 155-156, 2008. 
19. Vallon R, Freuler F, Desta-Tsedu N, et al: Serum amyloid A (apoSAA) expression is up-regulated in rheumatoid arthritis and induces transcription of matrix metalloproteinases. J Immunol 66: 2801-2807, 2001.

20. de Seny D, Cobraiville G, Charlier E, et al: Acute-phase serum amyloid a in osteoarthritis: regulatory mechanism and proinflammatory properties. PloS One 8: e66769, 2013.

21. Metawi SA, Abbas D, Kamal MM and Ibrahim MK: Serum and synovial fluid levels of interleukin-17 in correlation with disease activity in patients with RA. Clin Rheumatol 30: 1201-1207, 2011.

22. Migita K, Koga T, Torigoshi T, et al: Serum amyloid A protein stimulates CCL20 production in rheumatoid synoviocytes. Rheumatology (Oxford) 48: 741-747, 2009.

23. Mullan RH, Bresnihan B, Golden-Mason L, et al: Acute-phase serum amyloid A stimulation of angiogenesis, leukocyte recruitment, and matrix degradation in rheumatoid arthritis through an $\mathrm{NF}-\kappa \mathrm{B}$-dependent signal transduction pathway. Arthritis Rheum 54: 105-114, 2006.
24. Okamoto H, Katagiri Y, Kiire A, Momohara S and Kamatani N: Serum amyloid A activates nuclear factor-kappa B in rheumatoid synovial fibroblasts through binding to receptor of advanced glycation end-products. J Rheumatol 35: 752-756, 2008.

25. Farheen K and Agarwal SK: Assessment of disease activity and treatment outcomes in rheumatoid arthritis. J Manag Care Pharm 17 (9 Suppl B): S09-S13, 2011.

26. Connolly M, Mullan RH, McCormick J, et al: Acute-phase serum amyloid A regulates tumor necrosis factor $\alpha$ and matrix turnover and predicts disease progression in patients with inflammatory arthritis before and after biologic therapy. Arthritis Rheum 64: 1035-1045, 2012

27. Thorn CF, Lu ZY and Whitehead AS: Regulation of the human acute phase serum amyloid A genes by tumour necrosis factor-alpha, interleukin-6 and glucocorticoids in hepatic and epithelial cell lines. Scand J Immunol 59: 152-158, 2004.

28. Agrawal S, Misra R and Aggarwal A: Autoantibodies in rheumatoid arthritis: association with severity of disease in established RA. Clin Rheumatol 26: 201-204, 2007. 